\title{
Allometry and size control: What can studies of body size regulation teach us about the evolution of morphological scaling relationships?
}

\author{
Christen K. Mirtha, b* W. Anthony Frankino ${ }^{c^{*}}$, and Alexander W. Shingleton ${ }^{\mathrm{d}^{*}}$ \\ corresponding author: Christen K. Mirth (christen.mirth@monash.edu) \\ a Development, Evolution and the Environment Lab, Instituto Gulbenkian de \\ Ciência, Rua da Quinta Grande, 6, 2780-156 Oeiras, Portugal \\ b School of Biological Sciences, Monash University, Melbourne, Victoria 3800, \\ Australia \\ c Department of Biology and Biochemistry, University of Houston, Houston, TX, \\ U.S.A. \\ d Lake Forest College, 555 North Sheridan Road, Lake Forest, IL 60045, U.S.A.
}

short title: development and evolution of allometry

\begin{abstract}
The relationship between organ and body size, known as morphological allometry, has fascinated biologists for over a century because changes in allometry generate the vast diversity of organism shape. Nevertheless, progress has been limited in understanding the genetic mechanisms that regulate allometries and how these mechanisms evolve. This is perhaps because allometry is measured at the population level, however adult organ and body size depends on genetic background and the developmental environment of individuals. Recent findings have enhanced our understanding of how insects regulate their organ and body sizes in response to environmental conditions, particularly nutritional availability. We argue that merging these developmental insights with a population genetics approach will provide a powerful system for understanding the evolution of allometry.
\end{abstract}

\section{Introduction}

Individuals regulate the growth of their organs and body to ensure that the size of morphological traits match final body size, regardless of the environmentally-induced variation encountered during ontogeny [1]. The maintenance of relative organ size across body sizes can be visualized as a morphological scaling relationship for a population, species or other biological group (Fig 1A, B). Over a century of effort describing such scaling relationships has revealed that the same organs can vary considerably in relative size, producing, for example, gross differences in body shape among insect orders (Figure 1C), or variation in how shape changes with size within species (Figure 
1D). In fact, morphologies diversify by changing organ scaling [2,3]. Since scaling is generally tightly regulated within species, this leads to the question: how does genetic variation among taxa change scaling relationships?

Progress in understanding how scaling relationships evolve has been limited by a lack of information regarding how animals developmentally control organ/body size scaling developmentally. However, recent elucidation of the developmental processes that regulate organ and body growth, particularly from studies dissecting the molecular mechanisms that regulate growth with respect to nutrition, promises to fill this gap [4]. In this review, we discuss current information regarding the developmental processes controlling organ and body size in the context of morphological scaling. From this perspective, we propose a strategy to uncover how scaling relationships evolve.

\section{Evolution and allometry}

Morphological allometry, henceforth termed allometry, describes how organ size scales with the size of the body or its constituent parts. The relative size of organs can vary within an individual over developmental time, known as ontogenetic allometry, across conspecifics of the same developmental stage, called static allometry, or across species of the same stage, termed evolutionary allometry. Traditionally, allometries are modelled using the allometric equation: $\log (y)=\log (b)+\alpha \log (x)$, where $y$ is the size of a trait (e.g., leg size), $x$ is the size of another trait (typically body size), $\alpha$ is the allometric coefficient (the slope of the relationship), and $b$ is a constant (the intercept of the relationship) [5]. A proportional increase in organ size with trait size, a condition called isometry, occurs when $\alpha=1$; in this case large individuals are uniformly magnified versions of smaller ones. Shape changes with size when $\alpha \neq 1$. When $\alpha>1$, a condition called hyperallometry, $y$ becomes disproportionally large as $x$ increases. Examples of hyperallometric traits include the exaggerated secondary sexual characteristics that males use to compete for females, such as the horns of male rhinoceros beetles [6], the mandibles of male stag beetles (Figure 1C, D) [7], or the antlers of red deer [8]. When $\alpha<1$, a condition called hypoallometry, $y$ increases in size at a rate below that of $x$, becoming disproportionally small as $x$ increases. Hypoallometric traits include brain size in mammals [9] and male genital size in arthropods [10]. Variation among groups in $b$ reflect differences in organ size relative to the body (e.g., Figure 1A, B), but such relative differences will remain constant across body sizes if the groups share the same $\alpha$ (i.e., when the scaling relationships are parallel) [11].

Because static allometries describe how organs and the body scale, they capture the relationship between size and shape. Comparative work has revealed that the parameters of morphological scaling relationships (i.e., $b$ or $\alpha$ ) can vary considerably for different organs within the same species (Figure 1,2), or for the same organ among species (Figure 1). Evolutionary biologists have therefore come to recognize that changes in the parameters of scaling relationships are a central component of the evolution of morphology $[1,3,12]$.

Two controversies have emerged regarding the evolution of allometry. The first concerns the evolvability of the parameters of allometric relationships. Some hold that phylogenetic conservatism and long periods of evolutionary stasis in the slope of scaling relationships may indicate that this parameter is developmentally and evolutionarily constrained [13*]. In contrast, others argue 
that genetic manipulation and artificial selection experiments on organ size that change the allometric slope suggests they are evolutionary labile $\left[11,14^{*}\right]$.

The second controversy centres on the nature of the selective forces that change the slope and intercept of allometric relationships. Models addressing allometry evolution $[10,13,15-18]$ are sensitive to the biological assumptions they employ, and thus can generate very different predictions regarding how scaling relationships will respond to the same patterns of selection. This lack of consensus is heightened by artificial selection experiments that change the slope of a trait's allometric relationship with body size when there is direct selection on organ size $[19,20]$, but not when there is direct selection on the slope of the allometry [21].

These controversies may result from researcher's focus on the allometries themselves, which are group-level phenomena, rather than the actual targets of selection - the variation among individuals in the developmental-genetic mechanisms that control trait size, body size, and the relationship between the two. Work over the last decade has made great progress in identifying how organ $[18,22-25]$ and body size are regulated $\left[4,26,27^{* *}\right]$. An explicit integration of these findings into theories of allometry evolution is essential if we are to resolve these controversies and deepen our understanding of morphological evolution.

\section{The developmental physiology of size regulation}

In most insects, adults do not moult and their sclerotized bodies impede further growth. Thus, organ and body size are determined by growth during the larval and pupal stages. In holometabolous insects, most adult organs arise from populations of cells that are sequestered during embryogenesis, the imaginal tissues or discs, which grow, undergo patterning, and differentiate during the late larval instars and pupal stage. Final organ and body size are thus a function of their initial size at the beginning of growth, growth rate, and the duration of growth [28,29].

Variation in organ and body size arises from genetic differences among individuals and from developmental plasticity, i.e., developmental modifications induced by the environment. Much is known about how organ development responds to nutritional variation. For example, plasticity in ovary size in fruit fly (Drosophila melanogaster), as determined by ovariole number, results from changes in ovary growth rate and the rate of ovariole addition in response to change in access to nutrition in larvae [30]. In the tobacco hornworm, Manduca sexta, nutrition-induced differences in wing size result from changes in the rate and duration of wing disc growth during the larval and pupal periods [31].

The processes that generate variation in organ or body size within species via developmental plasticity overlap at least partially with those that generate fixed, genetic differences in organ and body size among and within species. For example, differences in ovary size between $D$. simulans and D. melanogaster arise from differences in ovary growth rate after the third instar [30]. In contrast, however, populations of $D$. melanogaster vary in ovary size due to differential allocation of ovarian somatic cells to alternate cell fates [32], a process not involved in regulating ovary size in response to nutrition in this species. Such findings have lead investigators to ask to what extent do the mechanisms that produce fixed, genetic differences in organ and body size share common pathways with the mechanisms that underlie developmental plasticity in size? 
Three hormones, the insulin-like peptides, the steroid moulting hormone ecdysone, and the sequiterpinoid hormone juvenile hormone (JH), have overlapping roles in regulating the rate and duration of growth, and hence are central to size regulation in insects [4]. The insulin-like peptides regulate growth rate and growth duration in response to nutritional availability, and potentially to other environmental signals, in most animals [33,34]. Consequently, levels of insulin signalling transduce nutritional variation into variation in body and organ growth rates in D. melanogaster, M. sexta, and the buckeye butterfly Precis coenia. Both ecdysone and $\mathrm{JH}$ are typically considered morphogenetic hormones, inducing key developmental transitions that influence organ and body size such as moulting and metamorphosis in insects, and their synthesis can be influenced by environmental conditions including access to nutrition [35-37].

Both ecdysone and JH have opposing effects on organ and body growth. In $D$. melanogaster, $M$. sexta and $P$. coenia, ecdysone supresses the growth rate of the body yet promotes growth of the wing imaginal discs [31,38-40*]. In $M$. sexta and $P$. coenia, ecdysone and insulin-like peptides interact synergistically to induce growth in the wing discs $[38,41]$. In addition, while JH induces growth of the body in $M$. sexta and D. melanogaster [ $\left.42^{*}, 43^{* *}\right]$, it represses the growth of imaginal tissues when insulin signalling is low due to starvation $[44,45]$. Such differential effects of these regulators on organ and body growth could have important impacts on the regulation, expression, and evolution of morphological allometries.

In addition, the same hormonal pathway can affect the growth of various organs to differing degrees. Although D. melanogaster wings scale isometrically with the body in response to nutrition, the genital arch is hypoallometric $[22,46]$ (Figure 2A). Thus, small males have proportionally larger genital structures than large males. This is because the genital disc responds less to starvation than the wing disc, as the genitals express less of Forkhead Box 0 , a negative regulator of insulin signalling [23]. This reduces the insulin-sensitivity of the genitals, such that insulin signalling remains high in the genital disc irrespective of nutritional conditions, whereas insulin signalling tracks nutrition in the wing disc (Figure 2A). In the rhinoceros beetle, Trypoxylus dichotomus, increased insulinsensitivity appears to induce hyperallometric growth of the male horn, since reduced insulin-signalling affects horn length more than it does wing or genital size [18]. Thus, the levels of insulin signalling within an organ and how that tracks whole-body nutritional status appear to be crucial determinants of its scaling relationship with the body.

In extreme cases, scaling relationships become non-linear with some insects switching between alternate scaling relationships according to environmental cues, a phenomenon known as polyphenism. For example, the migratory brown planthopper, Nilaparvata lugens, typically develops short forewings and stublike hindwings. However, under high densities differential expression of alternate forms of the Insulin Receptor in the wing induce the production of long fore- and hindwings for dispersal, changing the intercept of the wing-body size scaling relationship [ $\left.47^{* *}\right]$. Thus, the same pathway responsible for generating differences in scaling relationships between organs, such as the wing and genitals of $D$. melanogaster and the horns and wings of rhinocerous beetles, can also modify allometry within an organ, namely the wing, in planthoppers. 
There is some evidence that these mechanisms that regulate the effect of nutrition on organ size within species account for differences in organ size between species [30]. As discussed above, ovary size in D. melanogaster is regulated by developmental nutrition and this regulation occurs via the insulin signalling pathway [48**]. Intriguingly, insulin signalling is suppressed in the ovaries of $D$. sechellia and this correlates with a corresponding decrease in ovary size compared to D. melanogaster [48]. Thus the insulin signalling pathway may control both the plastic response of ovary size to changes in developmental nutrition, as well as differences in ovary size between species. Whether interspecific differences in insulin signalling account for differences in morphological scaling, that is relative as well as absolute organ size, is unclear.

\section{A developmental perspective on the evolution of allometry}

Static allometries are a population-level phenomenon; they are estimated empirically by fitting a function to groups of individuals spanning the full range of body and organ size. While utilitarian, this population-level approach potentially confounds genetic and environmental sources of variation in organ and body size among individuals. This matters, as it is the genetic variation among individuals in the mechanisms that regulate growth in response to the environment - e.g., allelic variation among individuals in the insulin signalling pathway genes - that will respond to selection on the scaling relationship itself. To understand how allometries evolve, studies of scaling need to move below the population level and focus on the individual variation in relative organ size across body sizes. This, however, presents a challenge for empiricists: individuals express only one adult size, and so fitting a function to describe the morphological phenotypes produced by an individual across the full range of body size - i.e., the scaling relationship for an individual genotype - would seem at the surface to be impossible.

However, the recent development of genome reference panels in $D$. melanogaster $[49,50]$ provides new tools to study the evolution of developmental plasticity and how it relates to the evolution of allometry. These panels consist of hundreds to thousands of fully-sequenced, isogenic lineages. Because individuals within each lineage share a single genotype, they can be reared at different nutrition levels to produce the full range of size phenotypes for each genotype, to which a scaling relationship can be fit. Characterizing the morphological scaling relationships of individual genotypes in this manner offers a means to quantify genetic variation in morphological scaling relationships, and can be used to conduct association mapping of the genetic underpinnings of phenotypic variation in scaling. A similar approach using clonal insects, like aphids, would provide a tractable alternative, although the genomic resources have yet to be developed.

Coupled with an in-depth understanding of the developmental mechanisms underlying organ and body size plasticity, this provides a powerful entryway into understanding how allometries evolve. As a hypothesis, variation among individuals in organ allometry could result from variation in the same pathways that generate differences between organ allometries within individuals (Figure 2B). If this were true, genotypes that proved to have the least plasticity in, for instance, wing/body size scaling might also show reduced variation in insulin signalling in their wings across nutritional conditions. The approach outlined 
above would provide a means of addressing the degree to which the signalling pathways that regulate plasticity in organ and body size contribute to the evolution of allometry. Furthermore, they might provide an inroad into predicting how allometries evolve.

\section{Acknowledgements}

We thank the editors, Leslie Pick and Cassandra Extavour, for inviting us to write this review. This work was supported by funding from the Fundação Calouste Gulbenkian to C.K.M, and the National Science Foundation to W.A.F. (IOS0920720) and A.W.S. (IOS-1256565 and IOS-0919855).

Figure 1: The diversity of insect shape results from changes in the relative size of body parts. Panel A) Scaling relationships fit to hypothetical populations of butterflies and planthoppers illustrate differences in the size of the forewing relative to the thorax, but a constant ratio of wing/thorax size across all body sizes. Panel B) Morphological diversity among insect orders is produced by changing the size of the wing relative to the body, as in Panel A. Images are scaled to the same thorax length (dashed white line), and the wings of wasps, flies, dragonflies, planthoppers, and butterflies (clockwise from top left) are highlighted in blue. Panel C) Scaling relationships fit to hypothetical populations of male (filled circles) and female (open circles) stag beetles. Mandible size increases disproportionally with thorax size in males, producing changes in overall shape as size increases. Conversely, mandible size increases proportionally with increasing thorax size in females and thus shape does not change with size. Panel D) Mandible size increases disproportionally with increasing thorax length (dashed white line) in male stag beetles, as shown in the scaling relationship in Panel $\mathrm{C}$.

Figure 2: Differences in scaling relationships between organs in the same individual and within organs across genotypes. Panel A) illustrates wing and genital allometries with respect to body size and shows how these relate to differences in insulin signalling between these organs in larvae exposed to different nutritional environments during development. In B), we hypothesize that genetic differences in wing allometry result from genotype-specific differences in insulin signalling during wing development in larvae exposed to different nutritional environments.

\section{References:}

1. Gould SJ: Allometry and size in ontogeny and phylogeny Biol Rev Camb Phil Soc 1966, 41:587-640.

2. Stern DL, Emlen DJ: The developmental basis for allometry in insects. Development 1999, 126:1091-1101.

3. Thompson DW: On Growth and Form edn 2nd Ed. Cambridge: Cambridge University Press; 1917. 
4. Nijhout HJ, Riddiford LM, Mirth CK, Shingleton AW, Suzuki Y, Callier V: The developmental control of size in insects. WIREs Developmental Biology 2014, 3:113-134.

5. Huxley JS, Tessier G: Terminology of relative growth. Nature 1936, 137:780781.

6. Kawano K: Horn and wing allometry and male dimorphism in giant rhinoceros beetles (Coleoptera: Scarabaeidae) of tropical Asia and America. Ann. Entomol. Soc. Am. 1995, 88:92-99.

7. Kawano K: Cost of evolving exaggerated mandibles in stag beetles (Coleoptera: Lucanindae). Ann. Entomol. Soc. Am. 1997, 90:453-461.

8. Kruuk LEB, Slate J, Pemberton JM, Brotherstone S, Guinness F, Clutton-Brock $T$ : Antler size in red deer: heritability and selection but no evolution. Evolution 2002, 56:1683-1695.

9. Koh I, Lee MS, Lee NJ, Park KW, Kim KH, Kim H, Rhyu IJ: Body size effect on brain volume in Korean youth. Neuroreport 2005, 16:2029-2032.

10. Eberhard WG: Static allometry and animal genitalia. Evolution 2009, 63:48-66.

11. Shingleton AW, Frankino WA: New perspectives on the evolution of exaggerated traits. Bioessays 2012, 35:100-107.

12. Huxley JS: Problems of Relative Growth. London: Methuen \& Co. Ltd.; 1932.

*13. Pelabon C, Firmat C, Bolstad GH, Voje KL, Houle D, Cassara J, Le Rouzic A, Hansen TF: Evolution of morphological allometry. Ann $N$ Y Acad Sci 2014, 1320:58-75.

This review addresses the concept that allometric relationships show low evolvability, thus constraining phenotypic diversification. The authors review the evidence for the evolution of allometry, suggesting that the slope of allometric relationships does appear constrained. They conclude that the understanding of how selection acts on allometry remains insufficient to resolve whether it truly acts as a constraint.

*14. Stillwell RC, Shingleton AW, Dworkin I, Frankino WA: Tipping the scales: Evolution of the allometric slope independent of average trait size. Evolution 2016.

In this article, Stillwell and co-authors use artificial selection to explore whether scaling relationships between the wing and body can evolve independently of trait size. They find after 17 generations of selection, the slope of the morphological scaling relationship does change independently of the bivariate mean, although the degree of change is small. They conclude that understanding how scaling relationships evolve requires a more in depth understanding of how individual genotypic and phenotypic variation relates to population-level patterns of allometry.

15. Bonduriansky R, Day T: The evolution of static allometry in sexually selected traits. Evolution 2003, 57:2450-2458.

16. Petrie M: Are all secondary sexual display structures positively allometric and, if so, why? Animal Behaviour 1992, 43:173-175.

17. Kodric-Brown A, Sibly RM, Brown JH: The allometry of ornaments and weapons. Proc Natl Acad Sci USA 2006, 103:8733-8738. 
18. Emlen DJ, Warren IA, Johns A, Dworkin I, Lavine LC: A mechanism of extreme growth and reliable signaling in sexually selected ornaments and weapons. Science 2012, 337:pp. 860-864.

19. Wilkinson GS: Artificial sexual selection alters allometry in the stalkeyed fly Cyrtodiopsis dalmanni (Diptera, Diopsidae). Genetical Research 1993, 62:213-222.

20. Robertson FW: Changing the relative size of the body parts of Drosophila by selection. Genetical Research 1962, 3:169-180.

21. Egset C, Hansen TF, Le Rouzic A, Bolstad GH, Rosenqvist G, Pélabon C: Artificial selection on allometry: change in elevation but not slope. J. Evol. Biol. 2012, 25:938-948.

22. Shingleton AW, Das J, Vinicius L, Stern DL: The temporal requirements for insulin signalling during development in Drosophila. PLoS Biol. 2005, 3:e289.

23. Tang HY, Smith-Caldas MSB, Driscoll MV, Salhadar S, Shingleton AW: FOXO regulates organ-specific phenotypic plasticity in Drosophila. PLoS Genetics 2011, 7:e1002373.

24. Shingleton AW, Tang HY: Plastic flies: the regulation and evolution of trait variability in Drosophila. Fly 2012, 6:147-152.

25. Cheng LY, Bailey AP, Leevers SJ, Ragan TJ, Driscoll PC, Gould AP: Anaplastic lymphoma kinase spare organ growth during nutrient restriction in Drosophila. Cell 2011, 146:435-447.

26. Mirth CK, Shingleton AW: Integrating body and organ size in Drosophila: recent advances and outstanding problems. Front. Endocrinol. 2012, 3:1-13.

**27. Lavine L, Gotoh H, Brent CS, Dworkin I, Emlen DJ: Exaggerated trait growth in insects. Annu Rev Entomol 2015, 60:453-472.

In this comprehensive review outlining the mechanisms of extreme trait growth, the authors identify four key pathways that lead to exaggerated trait growth: the sex determination pathway, the appendage patterning pathway, the ecdysone/JH pathways, and the insulin signalling/target of rapamycin pathway. Further, the authors postulate that the developmental mechanisms underlying extreme trait growth reflect the type of selection acting on a given trait.

28. Davidowitz G, D'Amico LJ, Nijhout HF: Critical weight in the development of insect body size. Evol. Dev. 2003, 5:188-197.

29. D'Amico LJ, Davidowitz G, Nijhout HF: The developmental and physiological basis of body size evolution in an insect. Proc. R. Soc. B 2001, 268:1589-1593.

30. Hodin J, Riddiford LM: Different mechanisms underlie phenotypic plasticity and interspecific variation for a reproductive character in Drosophilids (Insecta: Diptera). Evolution 2000, 54:1638-1653.

31. Nijhout HF, Grunert LW: The cellular and physiological mechanism of wing-body scaling in Manduca sexta. Science 2010, 330:1693-1695.

32. Green DA, Extavour CG: Convergent evolution of a reproductive trait through distinct developmental mechanisms in Drosophila.

Developmental Biology 2012, 372:120-130. 
33. Ikeya T, Galic M, Belawat P, Nairz K, Hafen E: Nutrient-dependent expression of insulin-like peptides from neuroendocrine cells in the CNS contributes to growth regulation in Drosophila. Curr. Biol. 2002, 12:1293-1300.

34. Géminard C, Rulifson EJ, Léopold P: Remote control of insulin secretion by fat cells in Drosophila. Cell Metabolism 2009, 10:199-207.

*35. Koyama T, Rodrigues MA, Athanasiadis A, Shingleton AW, Mirth CK: Nutritional control of body size through FoxO-Ultraspiracle mediated ecdysone biosynthesis. eLife 2014, 3:e03091.

This article identifies a molecular mechanism through which nutrition modifies the timing of the critical weight ecdysone peak in $D$.

melanogaster. In pre-critical weight larvae, a negative regulator of insulin signalling, FoxO, binds to a component of the ecdysone receptor, Ultraspiracle, in the prothoracic gland and represses ecdysone synthesis. With continued feeding, insulin signalling rises in the prothoracic gland causing FoxO to become displaced from the nucleus, thereby permitting ecdysone synthesis.

36. Tu MP, Yin CM, Tatar M: Mutations in insulin signaling pathway alter juvenile hormone synthesis in Drosophila melanogaster. Gen. Comp. Endocrinol. 2005, 142:347-356.

37. Suren-Castillo S, Abrisqueta M, Maestro JL: Fox0 inhibits juvenile hormone biosynthesis and vitellogenin production in the German cockroach. Insect Biochem Mol Biol 2012, 42:491-498.

38. Nijhout HF, Smith WA, Schachar I, Subramanian S, Tobler A, Grunert LW: The control of growth and differentiation of the wing imaginal disks of Manduca sexta. Dev. Biol. 2007, 302:569-576.

39. Parker NF, Shingleton AW: The coordination of growth among Drosophila organs in response to localized growth-perturbation. Dev. Biol. 2011, 357:318-325.

*40. Herboso L, Oliveira MM, Talamillo A, Pérez C, González M, Martín D, Sutherland JD, Shingleton AW, Mirth CK, Barrio R: Ecdysone promotes growth of imaginal discs through the regulation of Thor in $D$. melanogaster. Scientific Reports 2015, 5:12383.

Here, Herboso et al 2015 show that while increasing ecdysone reduces the growth of the body, it promotes growth in the wing imaginal discs and other imaginal tissues. In the wing, ecdysone exerts its action by modulating the activity of $T h o r / 4 E-B P$, a negative regulator of insulin signalling.

41. Nijhout HF, Grunert LW: Bombyxin is a growth factor for wing imaginal discks in Lepidoptera. Proc. Natl. Acad. Sci. USA 2002, 99:15446-15450.

*42. Mirth CK, Tang HY, Makohon-Moore SC, Salhadar S, Gokhale RH, Warner RD, Koyama T, Riddiford LM, Shingleton AW: Juvenile hormone regulates body size and perturbs insulin signaling in Drosophila. Proc Natl Acad Sci U S A 2014, 111:7018-7023.

This article demonstrates that JH regulates body size by modifying growth rates but not developmental timing in D. melanogaster. Further, it 
shows that $\mathrm{JH}$ regulates growth rates by modifying the activity of the insulin-signalling pathway and by altering ecdysone synthesis.

**43. Hatem NE, Wang Z, Nave KB, Koyama T, Suzuki Y: The role of juvenile hormone and insulin/TOR signaling in the growth of Manduca sexta. BMC Biology 2015, 13:44.

This study highlights the role of $\mathrm{JH}$ in regulating the balance between nutrition-dependent signalling and body-size monitoring to determine final body size in Manduca sexta. Using black mutant larvae, which bear a mutation known to reduce JH synthesis, the authors find that if they reduce both JH synthesis and Target of Rapamycin signalling, they increase developmental time without affecting growth rate relative to control.

44. Truman JW, Hiruma K, Allee JP, MacWhinnie SGB, Champlin DT, Riddiford $\mathrm{LM}$ : Juvenile hormone is required to couple imaginal disc formation with nutrition in insects. Science 2006, 312:1385-1388.

45. Koyama T, Syropyatova MO, Riddiford LM: Insulin/IGF signaling regulates the change in commitment in imaginal discs and primordia by overriding the effect of juvenile hormone. Dev. Biol. 2008, 324:258265.

46. Shingleton AW, Estep CM, Driscoll MV, Dworkin I: Many ways to be small: different environmental regulators of size generate distinct scaling relationships in Drosophila melanogaster. Proceedings of the Royal Society B: Biological Sciences 2009, 276:2625-2633.

**47. Xu H-J, Xue J, Lu B, Zhang X-C, Zhuo J-C, He S-F, Ma X-F, Jiang Y-Q, Fan H-W, $\mathrm{Xu}$ JY, et al.: Two insulin receptors determine alternative wing morphs in planthoppers. Nature 2015, 519:464-467.

In this work, the authors provide an in-depth study of the mechanism that results in alternative wing morphs in the planthoppers. They find that the difference in growth between long-winged and short-winged planthoppers results from the activity of either homodimeric insulin receptors, in the case of the long winged morph, or of a heterodimer of two insulin receptors in the short wing. Insulin receptor 2 acts to suppress insulin signalling, and hence wing growth, in short-winged animals.

**48. Green DA, Extavour CG: Insulin signalling underlies both plasticity and divergence of a reproductive trait in Drosophila. Proceedings of the Royal Society B: Biological Sciences 2014, 281:20132673-20132673. Here, Green and Extavour show that interspecific difference in ovariole number between $D$. sechellia and $D$. melanogaster arise from differences in the levels of insulin signalling. Further, they show that allelic variation in the insulin receptor accounts for some of this variation. Finally, they show that the evolution of substrate specialization in D. sechellia and $D$. erecta correlates with reduced plasticity in ovariole number.

49. Mackay TF, Richards S, Stone EA, Barbadilla A, J.F. A, al. e: The Drosophila melanogaster Genetic Reference Panel. Nature 2012, 482:173-178. 
50. King EG, Macdonald SJ, Long AD: Properties and power of the Drosophila Synthetic Population Resource for the routine dissection of complex traits. Genetics 2012, 191:935-949. 
Figure 1

A)

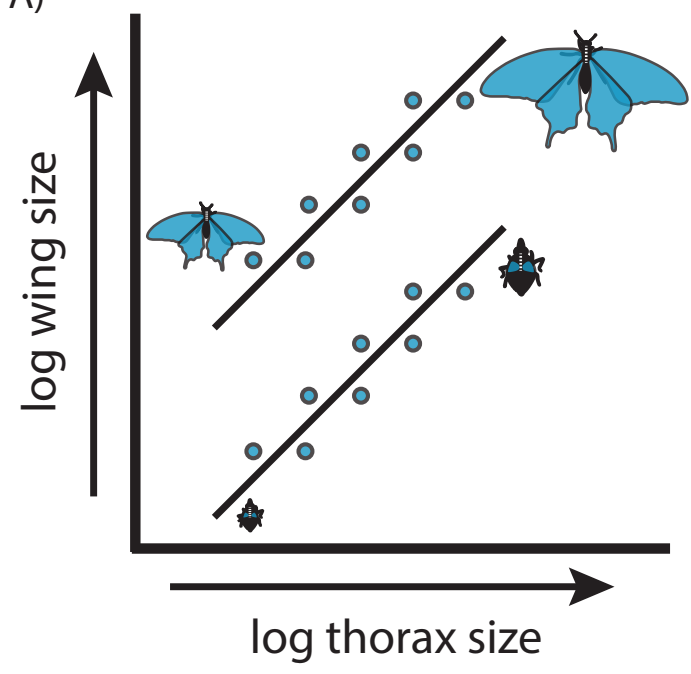

C)

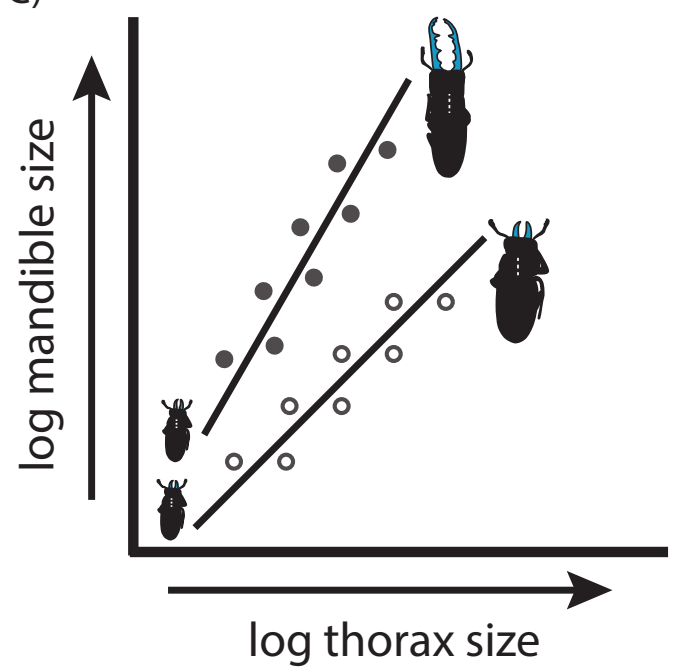

B)

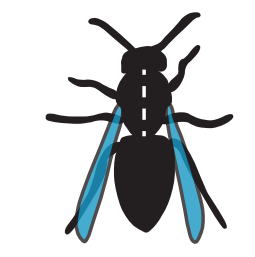

FIGURES

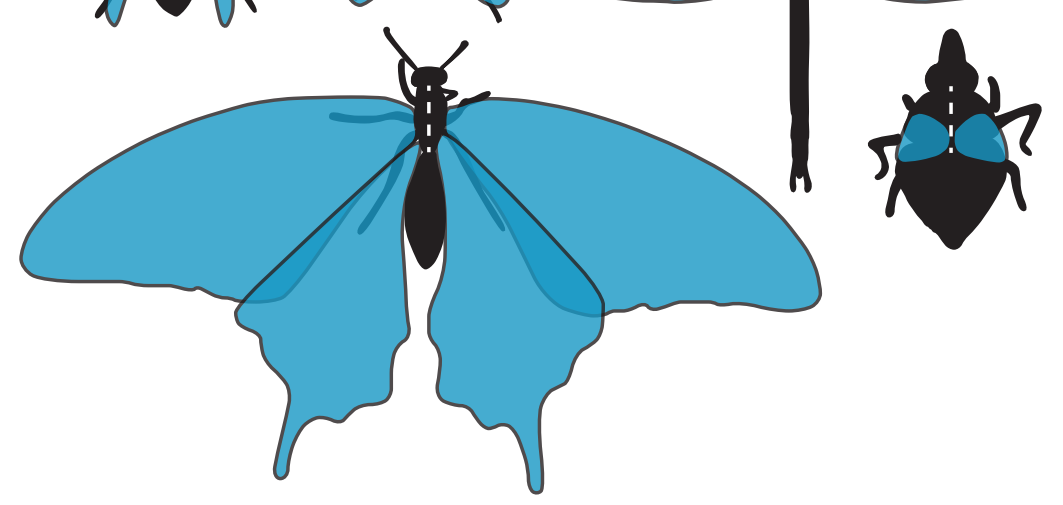

D)

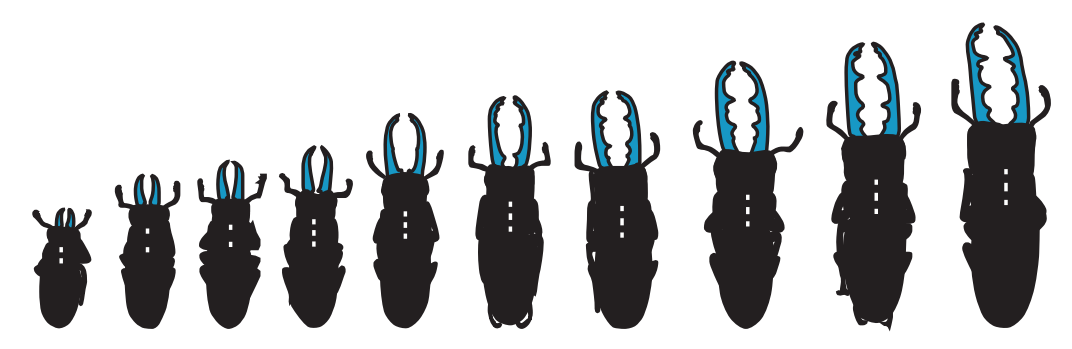


A)

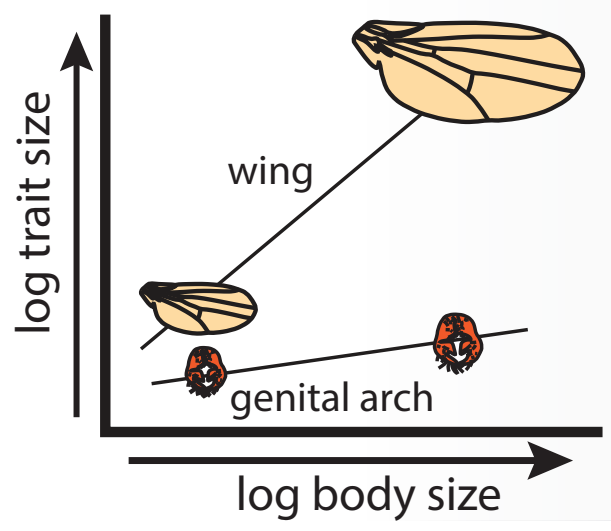

B)

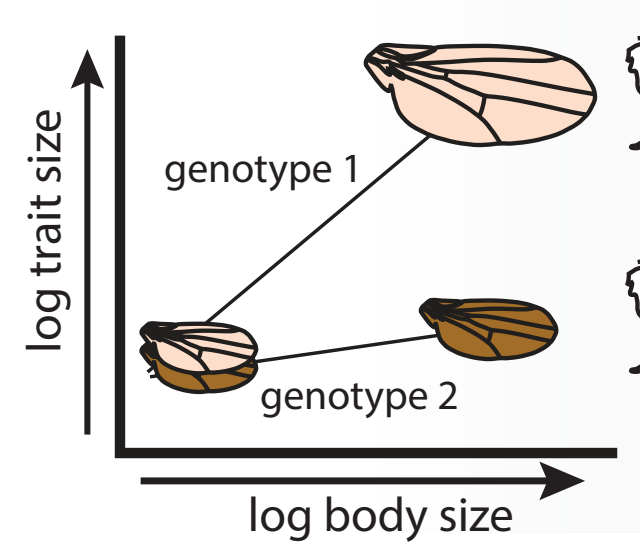

during development

high wing

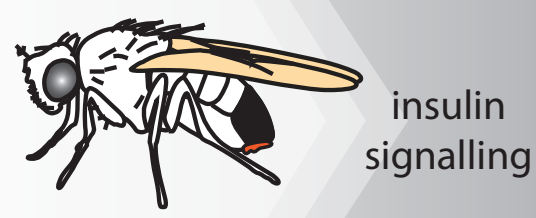

के

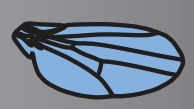

genital arch

(6)

?

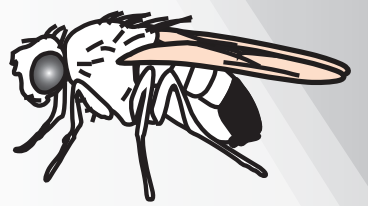

insulin signalling

\section{during development}

high genotype 1
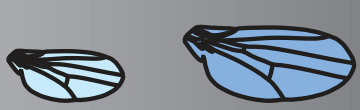

genotype 2

$\Rightarrow$ क

\section{nutrition}

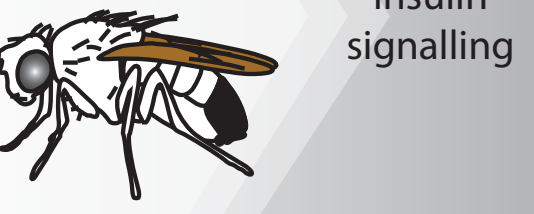

low 